\title{
Behandlung gutartiger Knochentumoren
}

\author{
Ulf Brunnemer, Christian Krettek, Thomas Gösling
}

\section{Zusammenfasssung}

Die benignen Knochentumoren werden in osteogene und knorpelbildende Tumoren sowie tumorähnliche Läsionen eingeteilt. Die in der Regel auf eine Lokalisation beschränkte Läsion bedarf einer differenzierten Diagnostik und einer von der Tumorentität, der Lokalität, der klinischen Symptomatik oder einer ossären Stabilitätsgefährdung abhängigen Therapie, die in der Regel operativ erfolgt, gelegentlich auch interventionell-radiologisch durchgeführt werden kann. Die häufigen „leave-me-alone-lesions“ bedürfen keiner weiteren therapeutischen Intervention.

\section{Therapy for Benign Bone Tumours}

Benign bone tumours are subdivided into osteogenic and cartilage-forming tumours as well as tumour-like lesions. The lesion which is usually limited to a single localisation requires a differentiated diagnostic procedure and treatment in accordance with the tumour entity, the location, the clinical symptoms and/or the risks of osseous instability. The therapy is usually surgical but can occasionally also be performed as an interventional radiological procedure. The frequent "leave me alone lesions" do not require any further treatment.

\section{Einleitung}

Benigne Knochentumoren werden nach der WHO-Klassifikation, Lyon 2002, in osteogene und knorpelbildende Tumoren sowie tumorähnliche Läsionen eingeteilt. Die verschiedenen Entitäten sind entsprechend ihrer histologischen Klassifikation in Tab. 1 zusammengefasst.

Statistisch verursachen Knochentumoren (benigne und maligne) ca. $1 \%$ aller neoplastischen Erkrankungen und stellen aufgrund des seltenen Auftretens die behandelnden Ärzte häufig vor diagnostische und therapeutische Probleme. Ein orthopädischer Chirurg ohne Spezialisierung auf muskuloskeletale Tumoren sieht statistisch in 3 Jahren durchschnittlich weniger als 1 primären Tumor des Skeletts. Die korrekte Unterscheidung in benigne und maligne Tumoren, die sich in ihrer Therapie gänzlich unterscheiden, bedarf einiger Erfahrung und sollte

OP-JOURNAL 2010; 26: 178-184

(c) Georg Thieme Verlag KG Stuttgart · New York DOI http://dx.doi.org/10.1055/s-0030-1250617 nach Möglichkeit in speziellen Zentren in interdisziplinärem Rahmen durchgeführt werden.

Benigne Knochentumoren stellen in den Häufigkeitsstatistiken von Dahlin [7] und Schajowicz [8], die aufgrund von Zahlen aus spezialisierten pathologischen Instituten erstellt worden sind und daher seltene Entitäten überrepräsentieren, rund $42 \%$ aller Knochentumoren dar.

Benigne Knochentumoren werden in osteogene und korpelbildende Tumoren sowie tumorähnliche Läsionen eingeteilt. Knochentumoren sind seltene Erkrankungen und die korrekte Diagnostik und Therapie bedarf Erfahrung und sollte in speziellen Zentren in interdisziplinärem Rahmen durchgeführt werden.

\section{Hauptteil}

Üblicherweise sind die gutartigen Knochentumoren auf eine Lokalität beschränkt, in sehr seltenen Fällen können sich jedoch ähnlich den malignen Tumoren biologisch wenig aggressive pulmonale Metastasen entwickeln, z.B. beim
Riesenzelltumor oder dem Chondroblastom. Das Überleben des Patienten wird hierdurch nicht negativ beeinflusst, eine analoge Situation ist z. B. für das benigne metastasierende Leiomyom des Uterus bekannt. Die gleiche Tumorentität kann sich lokal jedoch sehr unterschiedlich präsentieren.

\section{Diagnostik}

Die Diagnostik beinhaltet neben der Anamnese, körperlichen Untersuchung und Bildgebung ggf. Biopsie und Staging-Untersuchungen.

Da der überwiegende Anteil der gutartigen Knochentumoren klinisch stumm ist, treten sie häufig als Zufallsbefunde im Rahmen von Diagnostik anderer Intention auf (z. B. Röntgendiagnostik nach Trauma). Aggressiv wachsende Knochentumoren präsentieren hingegen häufig eine ausgeprägte klinische Symptomatik. Die primären Symptome sind palpable Schwellung und/oder progredienter Schmerz; klassischerweise besteht ein Ruhe- und Belastungsschmerz. Die bildgebende Diagnostik beinhaltet immer ein konventionelles Röntgenbild. Mithilfe des konventionellen Röntgens lassen sich einige Tumoren bereits mit hoher Wahrscheinlichkeit diagnostizieren, in anderen Fällen ist zumindest eine Eingrenzung auf einige Differenzialdiagnosen möglich. Bei Verdacht auf einen malignen Knochentumor gehört die Magnetresonanztomografie (MRT) zur zwingenden Diagnostik, da Weichteilkomponenten mit hoher Auflösung evaluiert werden können. Die Computertomografie (CT) bietet hingegen Vorteile in der Beurteilung der Tumormatrix, der Kortikalis und Spongiosa sowie der Periostreaktion. Die Skelettszintigrafie komplettiert die bildgebende Diagnostik der Knochentumoren.

Bei klinisch und radiologisch unklarem Befund ist eine Biopsie zur Klärung der Tumorentität bei nicht sicher auszu- 


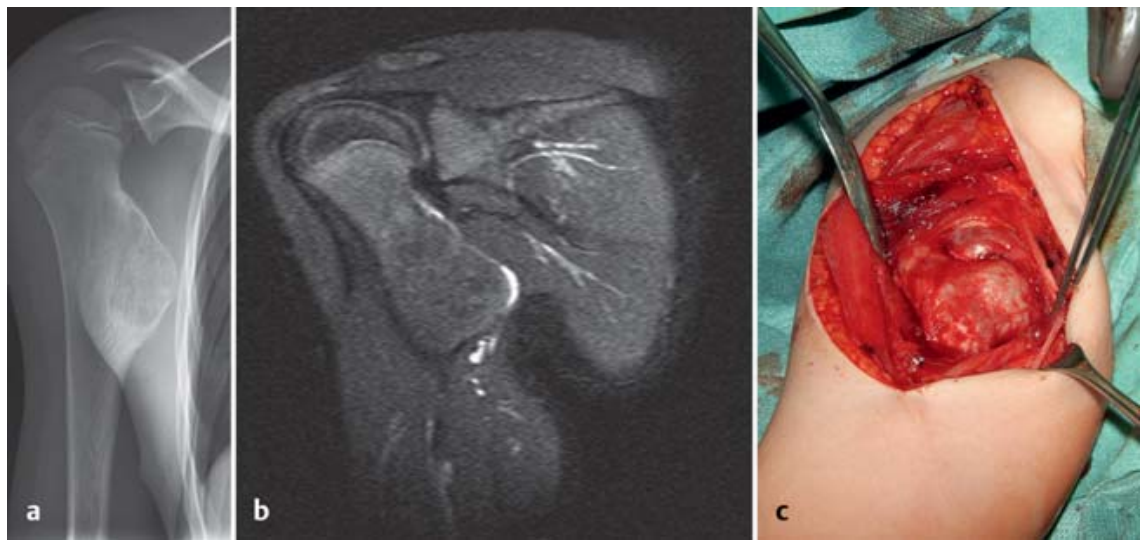

Abb. 1 a bis c Sessiles Osteochondrom proximaler Humerus: a konventionelles Röntgen; b MRT - Darstellung der Knorpelkappe; c intraoperativer Situs bei Resektion.

schließender Malignität indiziert. An dieser Stelle sei auf die Notwendigkeit einer korrekten Biopsietechnik verwiesen.

In Abhängigkeit von der Tumorentität schließt ein Staging mit CT des Thorax und Abdomens die Diagnostik der Knochentumoren ab. Bis auf wenige Ausnahmen (Riesenzelltumor, Chondroblastom) ist das Staging den malignen Knochentumoren vorenthalten.

\section{Therapie}

Die Notwendigkeit einer Therapie benigner Knochentumoren hängt von der Tumorentität, der Lokalität, der klinischen Symptomatik oder einer evtl. Stabilitätsgefährdung eines Knochens ab. Häufig sind gutartige Tumoren klinisch inapparent, Zufallsbefunde und bedürfen keiner konkreten Behandlung. In diesen Fällen wird häufig von einer sog. „leave-me-alone-lesion“ gesprochen.

Ist eine Behandlung benigner Knochentumoren notwendig, so erfolgt diese in der Regel operativ. In seltenen Fällen sind interventionell-radiologische Therapieverfahren, wie Radiofrequenzablation oder eine Strahlentherapie, für den Patienten schonender und mit kürzerer Rekonvaleszenz verbunden.

Die operative Therapie unterscheidet 4 Resektionsarten:

1. Intraläsional. Entfernung des Tumors von innen heraus in mehreren Stücken als Kürettage oder durch Verletzung der Tumorkapsel bei der Exzision. Eine komplette Resektion kann vom Pathologen nicht beurteilt werden. Sie kommt vorwiegend bei gutartigen Tumoren zur Anwendung, kann aber in bestimmten Fällen auch bei niedrig malignen Tumoren durchgeführt werden.

2. Marginal. Es erfolgt eine äußere Enbloc-Resektion entlang der (Pseudo-) Kapsel. Die Resektion verläuft durch die reaktive Zone. Der eigentliche Tumor wird ohne Eröffnung vollständig entfernt. Verbliebene Satelliten sind jedoch nicht auszuschließen. Bei hoch malignen Tumoren besteht eine große Gefahr des Lokalrezidivs. Die marginale Resektion kommt vorwiegend bei gutartigen Tumoren zur Anwendung, die entweder gut zugänglich sind (z.B. Osteochondrom) oder die aufgrund eines aggressiven Wachstums eine intraläsionale Resektion verbieten.

3. Weit. Der Tumor wird en bloc außerhalb der reaktiven Zone im gesunden Gewebe innerhalb eines Kompartiments reseziert. Der gesamte Tumor ist von gesundem Gewebe umgeben. Der Resektionsabstand innerhalb des Kompartiments soll zwischen 2 und $4 \mathrm{~cm}$ liegen. Es besteht bei der weiten Resektion das Risiko von Skip-Metastasen innerhalb des Kompartiments.

4. Radikal. Der Tumor wird en bloc unter Mitnahme der betroffenen Kompartimente reseziert. Bei unversehrten Kompartmentgrenzen (Kortikalis, Faszie) ist der Abstand zum Tumor hier von untergeordneter Bedeutung. Ist der Tumor z.B. von einer intakten Kompartmentfaszie abgegrenzt, ist dieser Resektionsrand als radikal zu bewerten.

Weite und radikale Tumorresektionen finden in der Therapie maligner Knochentumoren Anwendung, benigne Knochentumoren werden i.d. R. intraläsional oder marginal reseziert.

Der Vollständigkeit nach sei erwähnt, dass die Therapie der malignen Knochen-
Tab. 1 Benigne Knochentumoren.

\begin{tabular}{|c|c|}
\hline & benigne Tumorentitäten \\
\hline $\begin{array}{l}\text { osteogene } \\
\text { Tumoren }\end{array}$ & $\begin{array}{l}\text { Osteom/Enostom } \\
\text { Osteoidosteom } \\
\text { Osteoblastom }\end{array}$ \\
\hline $\begin{array}{l}\text { knorpel- } \\
\text { bildende } \\
\text { Tumoren }\end{array}$ & $\begin{array}{l}\text { Chondrom } \\
\text { - Enchondrom } \\
\text { - periostales (juxtakortika- } \\
\text { les) Chondrom } \\
\text { Osteochondrom (kartilagi- } \\
\text { näre Exostose) } \\
\text { Chondroblastom } \\
\text { Chondromyxoidfibrom }\end{array}$ \\
\hline $\begin{array}{l}\text { Riesen- } \\
\text { zelltumor }\end{array}$ & Osteoklastom \\
\hline $\begin{array}{l}\text { tumor- } \\
\text { ähnliche } \\
\text { Läsionen }\end{array}$ & $\begin{array}{l}\text { fibröser metaphysärer Defekt } \\
\text { (nicht ossifzierendes Fibrom) } \\
\text { fibröse Dysplasie } \\
\text { osteofibröse Dysplasie } \\
\text { solitäre Knochenzyste } \\
\text { aneurysmatische Knochen- } \\
\text { zyste }\end{array}$ \\
\hline
\end{tabular}

tumoren neben der Resektion durch den Chirurgen interdisziplinär durch Onkologen und Strahlentherapeuten erfolgt.

\section{Adjuvante lokale Therapie}

Um bei intraläsionaler Resektion von aggressiven Läsionen den chirurgischen Rand zu vergrößern, existiert eine Reihe an adjuvanten lokalen Maßnahmen. In der Literatur finden sich hierzu Empfehlungen für Ethanol, Phenol und Wasserstoffperoxid. Die Kryotherapie mit flüssigem Stickstoff ist ein weiteres Verfahren. PMMA („polymethyl methacrylate“) soll durch die Hitzeentwicklung beim Aushärten ebenfalls einen positiven Effekt auf den Resektionsrand besitzen. Es muss jedoch klar sein, dass es keinen Nachweis und somit keine Sicherheit für eine Erweiterung des Resektionsrands gibt.

\section{Rekonstruktionsmöglichkeiten nach Resektion}

Bei jeder Resektion, ob intraläsional oder radikal, verbleibt ein knöcherner Defekt, welcher in der Regel eine ossäre Rekonstruktion notwendig macht. Es bieten sich unterschiedliche Rekonstruktionsmöglichkeiten an:

1. biologische Rekonstruktion, bspw. durch Autografts, Allografts oder Kallusdistraktion

2. alloplastischer Ersatz durch Tumorendoprothetik oder die Implantation von Knochenzement 
Biologische Rekonstruktionen bieten zahlreiche Vorteile, so zeichnen sie sich nach ossärer Integration durch eine lebenslange Haltbarkeit aus und sollten nach Möglichkeit Anwendung finden. In einigen Ausnahmen kann aufgrund der verbliebenen Stabilität auf eine ossäre Rekonstruktion verzichtet werden. Bei lediglich temporär geschwächten Knochen kann jedoch eine temporäre Osteosynthese, Ruhigstellung oder zumindest Entlastung notwendig sein.

\section{Tumorentitäten und spezielle Behandlungsstrategien}

\section{Osteogene Tumoren}

\section{Osteom/Enostom}

Das Osteom ist klinisch und radiologisch eine juxta- oder intraossäre Knochenverdichtung im Sinne einer umschriebenen Hyperostose und besteht histologisch aus normalem Knochengewebe und ist daher nicht als neoplastische Erkrankung zu werten. Aus diesem Grund listen die Initiatoren der Lyon-WHO-Klassifikation das Osteom nicht mehr unter die Knochentumoren; zur Vollständigkeit sei es an dieser Stelle erwähnt. Das Osteom ist als reaktiv-reparative Knochenneubildung zu werten, wie bspw. nach einem Trauma, nach einer Infektion oder als Antwort auf eine mechanische Reizung, z.B. durch einen Tumor oder ein Knochenimplantat, Stromaossifikationen, z.B. von Metastasen, oder auch als solide Matrixossifikation, z.B. nach länger bestehenden Knorpelläsionen. Die Osteome werden in 3 Gruppen eingeteilt: 1. klassisches Osteom (Elfenbeinexostose) - fast ausschließlich vorkommend am Schädelknochen; 2. juxtakortikales (paraossales) Osteom, welches meist an langen Röhrenknochen beobachtet wird; 3. medulläres Osteom, das sog. Enostom, welches eine Kompaktainsel im spongiösen Knochen darstellt.

Osteome sind als Normvariante zu werten, da sie bei ca. $10 \%$ der Patienten vorkommen und sich meist asymptomatisch darstellen. Am Stammskelett und den Extremitäten ist das Osteom normalerweise als „leave-me-alone-lesion“ zu werten. Eine therapeutische Konsequenz ergibt sich lediglich bei klinischer Symptomatik. Am Schädel kommt es gelegentlich zu einer Verlegung der Ostien der Nasennebenhöhlen mit konsekutivem Sekretaufstau und Kopfschmerzen, oder generell zur Kompression den Knochen anliegender Strukturen wie Nerven, Gefäße oder Sehnen.

\section{Osteoidosteom}

Das Osteoidosteom ist relativ häufig (ca. $4 \%$ aller primären Knochentumoren). Es besteht aus einem kleinen Nidus, der von einer dicken Schicht reaktiven reifen Knochens umgeben ist. Es liegt typischerweise intrakortikal. Der Nidus besteht aus hochvaskularisiertem Gewebe mit Osteoid, er zeigt sich im konventionellen Röntgenbild strahlendurchlässig. Zentral kann sich eine Sklerosezone abbilden. Die Läsion findet sich typisch im Stadium 2 und wird vorwiegend im Wachstumsalter oder jungen Erwachsenenalter diagnostiziert. Spontane Heilungen sind beschrieben. Das Osteoidosteom zeigt kein expansives Wachstum und ist kaum größer als $1 \mathrm{~cm}$. Selten zeigt das Osteoidosteom mehr als $1 \mathrm{Ni}$ dus. Klinisch berichten die Patienten über einen plötzlich einsetzenden, gut lokalisierbaren Schmerz, der eher bei Ruhe oder in der Nacht als bei Belastung auftritt. Typisch ist das gute Ansprechen auf Salizylate. Das Osteoidosteom entgeht gelegentlich aufgrund seiner Größe und Lage der konventionellen Röntgendiagnostik. Die Szintigrafie ist hier hochsensitiv. Die höchste Spezifität erreicht die Dünnschicht-CT. Die Kombination aus typischer Klinik und Bildgebung ist zielgerichtet. Differenzialdiagnostisch ist der Brodie-Abszess in Betracht zu ziehen.

Auch wenn das Osteoidosteom spontan abheilen kann, sollte bei entsprechender Klinik die Entfernung erfolgen. Bei intraläsionaler Kürettage liegt das Rezidivrisiko unter 5\%. Problematisch ist häufig das intraoperative Auffinden des Nidus mit einem angemessenen Zugang. Als Alternative steht hier die CT-gesteuerte Radiofrequenzablation zur Verfügung. Ist diese aufgrund der Nähe zu gefährdeten Strukturen nicht möglich, kann die CTgesteuerte Drahtmarkierung oder die Navigation hilfreich sein. Das Überbohren des Nidus stellt ebenfalls eine Alternative dar.

\section{Osteoblastom}

Das Osteoblastom wird häufig als „großer Bruder" des Osteoidosteoms bezeichnet. Die Bezeichnung ist jedoch irreführend, da das klinische und radiologische Bild differieren. Das Osteoblastom findet sich am häufigsten in den dorsalen knöchernen Abschnitten der Wirbelsäule, bevorzugt bei Männern zwischen dem 15. und 30. Lebensjahr. Es wird am häufigsten im Stadium 2 als langsam wachsende, schmerzhafte Läsion gefunden. Eine Skoliose kann Reaktion auf ein schmerzhaftes Osteoblastom sein. Selten präsentiert

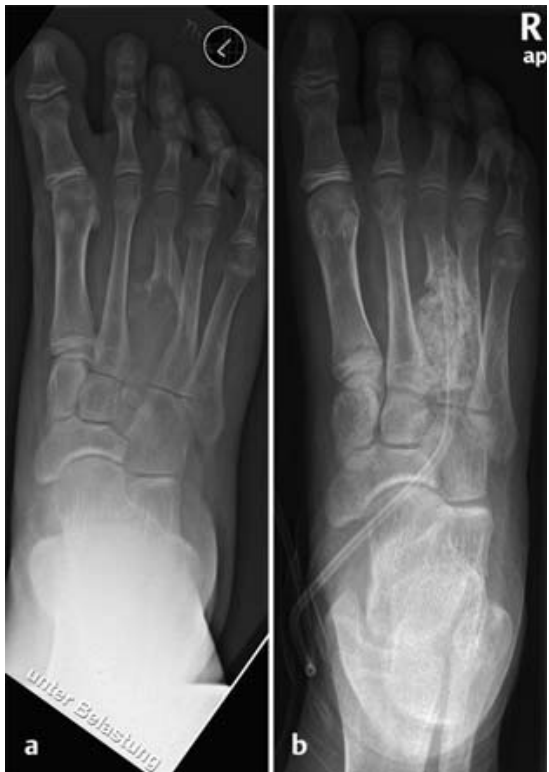

Abb. 2a und b Aneurysmatische Knochenzyste am Os metatarsale III: a präoperativ; b nach intraläsionaler Kürettage und Defektauffüllung mit allogener Spongiosa.

sich das Osteoblastom im Stadium 3 mit pathologischer Fraktur und sekundärer aneurysmatischer Knochenzyste. Der Tumor ist typischerweise über $2 \mathrm{~cm}$ groß und zeigt sich sphärisch oder oval. Das Osteoblastom zeigt in der Regel eine geringere reaktive Sklerose als das Osteoidosteom. Expansives Wachstum (sog. „blow out“) ähnlich der aneurysmatischen Knochenzyste ist ebenfalls beschrieben. Im aggressiven Stadium kann es einem malignen Tumor ähneln. Histopathologisch zeigt sich ein Befund ähnlich dem Osteoidosteom bei jedoch größerer Neigung zur Ausbildung einer sekundären aneurysmatischen Knochenzyste. Als Sonderform können aggressive Osteoblastome mit „epitheloiden“ vergrößerten Osteoblasten auftreten. Selten finden sich Osteoblastome im Bereich der langen Röhrenknochen.

Die intraläsionale Resektion führt in Stadium 2 zu ca. 20\% Rezidivrate, während im Stadium 3 bis zu 50\% Rezidive beschrieben sind. Die En-bloc-Resektion (marginal oder im Stadium 3 besser weit) gewährleistet die geringste Rezidivrate, muss aber mit der funktionellen Einschränkung bei dieser nicht malignen Erkrankung abgewogen werden (Abb. 3).

Knorpelbildende Tumoren

\section{Osteochondrom}

Das Osteochondrom ist der häufigste gutartige Knochentumor. Synonym wird der ältere Begriff der kartilaginären Exo- 


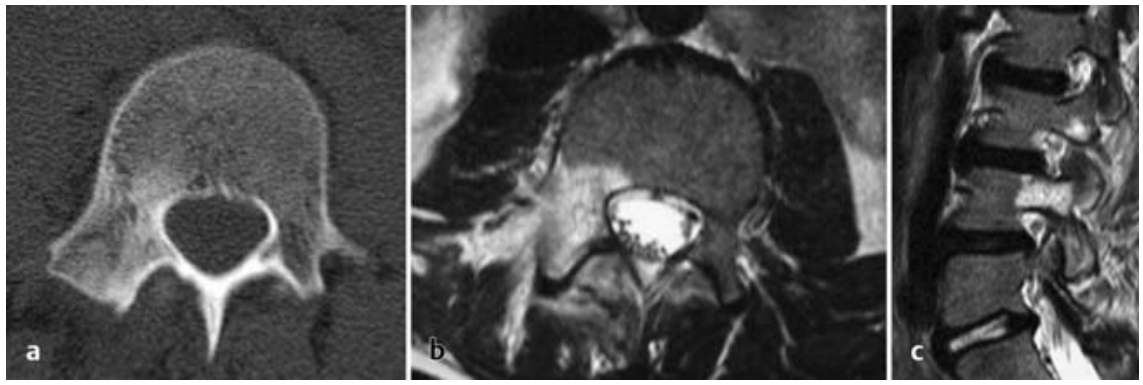

Abb. 3 a bis c Osteoblastom: Osteoblastom im rechten Pedikel LWK 4: a CT axial; b MRT axial; c MRT sagittal.

stose verwendet. Das Osteochondrom ist charakterisiert als eine knöcherne Ausziehung mit einer oberflächlichen Knorpelkappe, die in ihrem Aufbau der Wachstumsfuge gleicht. Der ossäre Anteil kann gestielt (pedikulär) oder breitbasig (sessil) imponieren. Das Osteochondrom findet sich vornehmlich metaphysär im Bereich des Kniegelenks und des proximalen Humerus. Weniger häufig findet man es im Bereich der Skapula, des Beckens, des proximalen Femurs oder der distalen Tibia. Die spontane Rückbildung eines Osteochondroms ist lediglich sporadisch beschrieben. Osteochondrome werden i.d.R. in der 1. und 2. Dekade des Lebens diagnostiziert. Mit dem Wachstum kommt es häufig im Verlauf zu einer Größenzunahme des lokalen Befunds. Nach Abschluss des Größenwachstums persistiert auch das Wachstum des Osteochondroms. Das Osteochondrom für sich ist asymptomatisch. Schmerzen können durch Druck auf Muskeln, Nerven, benachbarte Knochen oder Gefäße auftreten. Die maligne Transformation eines solitären Osteochondroms tritt in weniger als $1 \%$ der Fälle ein und rechtfertigt nicht die prophylaktische Entfernung eines asymptomatischen Befundes. Neu aufgetretene Schmerzen und/oder Größenzunahme nach dem Wachstumsabschluss sollten jedoch an die Möglichkeit einer sekundären Entartung denken lassen.

Das Osteochondrom lässt sich bereits im konventionellen Röntgenbild eindeutig zuordnen. Das gestielte Osteochondrom wächst meist von der Wachtumsfuge weg. Typisch ist der kontinuierliche Übergang der Kortikalis des Ursprungsknochens in den Kortex des Osteochondroms. Der spongiöse zentrale Anteil geht kontinuierlich in den Markraum über. Die Knorpelkappe kann einzelne Ossifikationen zeigen. Im MRT lässt sich die Knorpelkappe am besten darstellen. Eine Dicke von über $20 \mathrm{~mm}$ wird als kritisch für das Risiko einer malignen Trans- formation gesehen. Alternativ kann für den Geübten die Knorpelkappe gut in der Sonografie dargestellt werden.

Multiple hereditäre Osteochondrome, auch Exostosenkrankheit genannt, werden autosomal dominant mit inkompletter Ausprägung beim weiblichen Geschlecht vererbt. Die multiplen Osteochondrome können mit einem Fehlwachstum im Bereich der langen Röhrenknochen einhergehen. Die maligne Transformation zum Chondrosarkom ist bei den multiplen Osteochondromen höher.

Symptomatische Osteochondrome sollten reseziert werden. Es empfiehlt sich, eine präoperative Schnittbilddiagnostik durchzuführen, um eine genaue Lagebeziehung zu den Gefäßen und Nerven darzustellen. Das Osteochondrom kann marginal reseziert werden. Im Bereich der Basis sollte bei verbliebenen Knorpelresten noch einmal nachkürettiert werden. Bestehen Zweifel bez. der Dignität, können kleine Osteochondrome als Exzisionsbiopsie marginal entfernt werden. Hierbei sollte sich der Zugang Biopsie ausrichten.

\section{Enchondrom}

Das Enchondrom ist der zweithäufigste gutartige Knochentumor. Seine Matrix besteht aus reifem hyalinen Knorpel, welche das Chondrom charakterisiert. Das Chondrom geht auf dysplastische Chondrozyten der Epiphysenfuge zurück, die nicht den gewohnten Weg der enchondralen Ossifikationen gehen. Das Enchondrom liegt im Gegensatz zum periostalen oder juxtakortikalen Chondrom innerhalb des Knochens. In der Regel findet sich eine zentrale Lage. Die Röhrenknochen der Hand sind die häufigste Lokalisation, gefolgt vom Femur, dem proximalen Humerus, der Tibia und den Rippen. Die meisten Enchondrome werden als Zufallsbefund gefunden. Selten an den allgemeinen Empfehlungen zur sind sie für eine pathologische Fraktur (hier überwiegend im Bereich der Hand) verantwortlich. In der konventionellen Röntgendiagnostik zeigt sich das Enchondrom im Bereich der Hand und des Fußes typisch als Aufhellung ohne scharfe Demarkierung. In den langen Röhrenknochen hingegen zeigt das Enchondrom charakteristische Kalzifizierungen („rings and arcs“), die ein popcornartiges Bild ergeben. Der Kortex kann bei entsprechender Größe ausgedünnt sein. Das Enchondrom ist während des Wachstums aktiv und befindet sich im Stadium 2. Nach Abschluss des Wachstums befindet sich das Enchondrom im Stadium 1 ohne Größenzunahme. Eine Größenzunahme nach Abschluss des Wachstums ist verdächtig auf eine maligne Transformation. Multiple Enchondrome kommen im Rahmen des Morbus Ollier vor. Hier ist die Verteilung der multiplen Enchondrome unilateral. Wachstumsstörungen insbesondere im Bereich des Radius und der Tibia sind beschrieben. Das Maffuci-Syndrom beinhaltet zu den multiplen Enchondromen das Vorhandensein von kutanen Hämangiomen.

Die wichtigste Differenzialdiagnose zum Enchondrom stellt das Chondrosarkom und hier v.a. das hochdifferenzierte Chondrosarkom (G1) dar. Dies gilt insbesondere für die großen, markraumausfüllenden Läsionen. Folgende Zeichen sollten den Verdacht auf ein Chondrosarkom lenken:

- signifikante Größenzunahme nach Wachstumsabschluss,

- Ruhe- und Nachtschmerz,

- lokalisierte Verdickung der Kortikalis,

- Ausdünnung der inneren Kortikalis um mehr als $2 / 3$,

- Destruktion der Kortikalis mit Einbruch in die Weichteile,

- Periostreaktion.

Die CT ist als Schnittbildverfahren hier dem MRT zu bevorzugen. Die Größe der Läsion wird kontrovers diskutiert. Die maligne Transformation einer solitären Läsion ist sehr unwahrscheinlich (unter $1 \%)$, steigt bei Vorliegen eines multiplen Befalls aber auf ca. 10\%. Es gilt, dass stammnahe Läsionen eher $\mathrm{zu}$ einer Transformation neigen. Das Prädilektionsalter der malignen Transformation liegt zwischen 30 und 50 Jahren. Die Patienten müssen über das Risiko einer malignen Transformation aufgeklärt werden. Eine prophylaktische Entfernung ist nicht empfohlen. Ebenso ist die Biopsie ohne das Vorliegen eines Malignitätverdachts nicht angezeigt, da diese 


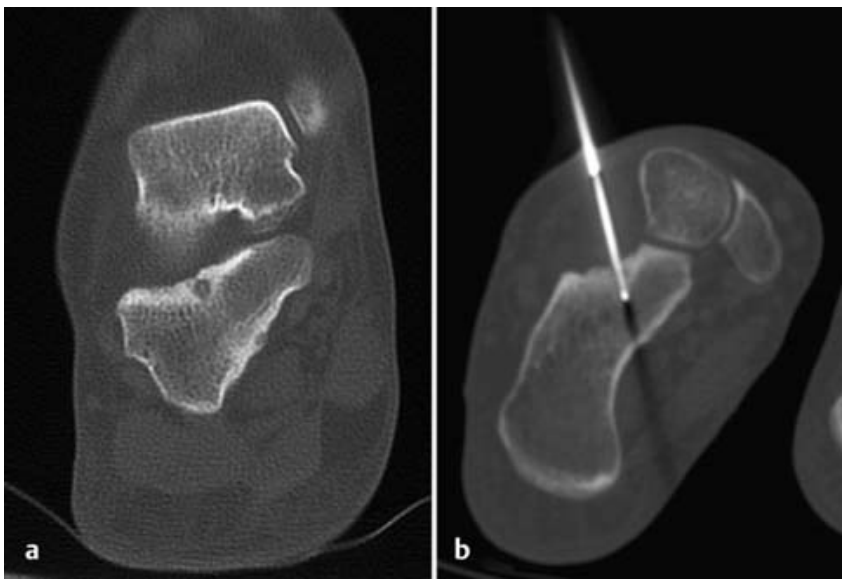

lediglich bei kompletter Kürettage Sicherheit bez. des Befunds erbringt. Liegen keine Malignitätskriterien im Röntgenbild vor, empfiehlt sich die konventionell radiologische und klinische Verlaufskontrolle in 6 Monaten. Auch bei möglich malignem Geschehen kann durchaus eine erneute Kontrolle (dann aber mit CT) gerechtfertigt sein, da das differenzialdiagnostische, hochdifferenzierte Chondrosarkom ein langsames Wachstum zeigt und in der Regel nicht metastasiert. Bei Stabilitätsgefährdung durch das Enchondrom ist eine intraläsionale Resektion durch Kürettage mit Spongiosaauffüllung möglich. Besteht der dringende Verdacht auf eine maligne Transformation, sollte eine Biopsie erfolgen. Sie kann beim differenzialdiagnostischen G1-Sarkom auch als Exzisionsbiopsie erfolgen. Diese muss sich jedoch nach den allgemeinen Regeln der Biopsie von Knochentumoren richten (s.o.)

\section{Chondroblastom}

Das Chondroblastom, auch Codman-Tumor genannt, hat seinen Ursprung fast ausnahmslos in der Epiphyse. Die häufigsten Lokalisationen sind das distale Femur, die proximale Tibia und der proximale Humerus. Das Chondroblastom zeigt sich häufig im Stadium 2 mit Schmerzen, kann sich aber auch sehr aggressiv mit expansivem Wachstum und Weichteilkomponente zeigen. Das Erscheinungsbild kann hier einem malignen Tumor ähneln. Durch die Nähe zum Gelenk zeigen ca. ein Drittel der Patienten einen Erguss. Das Alter bei Diagnosestellung liegt in der Regel zwischen 10 und 20 Jahren. Jungen sind häufiger betroffen. Radiologisch zeigt sich eine landkartenartige, überwiegend exzentrisch gelegene Aufhellung in der Epiphyse. Sie besitzt einen scharfen Rand, gelegentlich mit einer hauchdünnen Sklerose. Kalzifi-
Abb. $4 a$ und $b$ Osteidosteom im Kalkaneus subtalar: a CT koronar; b Therapie mittels CT-gesteuerter Punktion und Radiofrequenzablation.

zierungen als kleine, feine, „salz- und pfefferartige" Flecken werden beobachtet. Im aggressiven Stadium ist die Kortikalis durchbrochen. Die Behandlung des Chondroblastoms richtet sich nach dem Stadium. Im Stadium 2 liegt die Rezidivrate bei intraläsionaler Kürettage bei $10 \%$, welche bei deutlich funktionellem Vorteil in Kauf genommen werden sollte. Eine Kontamination des Gelenkkompartiments sollte jedoch nach Möglichkeit vermieden werden. Im Stadium 3 liegt die Rezidivrate bei intraläsionaler Resektion bei 50\%. Lokale Kontrolle ist lediglich durch eine weite Resektion gesichert. Bei der Entscheidung sind Funktionalität und das Risiko des Lokalrezidivs miteinander abzuwägen. Die Transformation zum Chondrosarkom ist sehr selten und vornehmlich im Stammbereich.

\section{Chondromyxoidfibrom}

Das Chondromyxoidfibrom tritt klassischerweise an den Metaphysen der langen Röhrenknochen der unteren Extremität sowie dem Talus auf. Im konventionellen Röntgenbild zeigt sich eine glatt berandete, randsklerosierte Struktur mit landkartenartiger Destruktion im Sinne einer ovalären Osteolyse, normalerweise ohne Matrixverkalkungen. Im MRT zeigt sich in den kontrastmittelgesättigten Sequenzen ein inhomogenes Kontrastmittelenhancement. Der hohe fibröse Anteil ergibt in der T1-Wichtung häufig ein muskelisointenses Signalverhalten, in den T2-Wichtungen eher eine hypointense Darstellung. Histologisch ist der Tumor teils chondroid, teils myxoid aufgebaut. Bei fehlendem Sklerosesaum oder Kortikalisperforation muss differenzialdiagnostisch immer an einen Riesenzelltumor gedacht werden. Die Therapie des Chondromyxoidfibroms stellt nach Möglichkeit die Exzision weit im Gesunden oder eine En-bloc-Resektion dar.
Eine alleinige intraläsionale Resektion durch Kürettage geht mit einer Rezidivquote von 12,5-25\% einher. Prinzipiell besteht die Möglichkeit einer malignen Entartung, diese ist jedoch extrem selten.

Tumorähnliche Läsionen

\section{Nicht ossifizierendes Fibrom/} Metaphysärer fibröser kortikaler Defekt Beide Entitäten sind histologisch identisch und stellen zusammen die häufigste fibröse Läsion des Knochens dar. Es handelt sich nicht um ein Neoplasma, sondern um einen hamartomatösen Defekt des Knochens. Die Läsionen finden sich vorwiegend in der Kindheit und dem jungen Erwachsenenalter mit einer Prävalenz von 30\%. Der fibröse, kortikale Defekt beschränkt sich auf die Kortikalis. Weitet sich der Prozess medullär aus, wird vom nicht ossifizierenden Fibrom (NOF) gesprochen. Das männliche Geschlecht ist bevorzugt. Die typische Prädilektionslokalisation ist der metaphysäre Kortex des distalen Femurs und der proximalen und distalen Tibia. Seltener sind der proximale Humerus oder die Fibula betroffen. Das Achsskelett und die Knochen von Hand und Fuß sind nicht betroffen. Die meisten NOFs sind Zufallsbefunde und klinisch stumm. In sehr seltenen Fällen kommt es zu einer pathologischen Fraktur. Das klassische NOF zeigt sich im konventionellen Röntgenbild als eine elliptische, strahlentransparente, häufig weintraubenartige Läsion mit direktem Bezug zur Kortikalis. Es findet sich eine scharfe sklerotische Abgrenzung. Die ovaläre Struktur richtet sich in Längsrichtung zur Schaftachse aus. Das konventionelle Röntgenbild ist richtungsführend. Das NOF kann im Verlauf des Wachstums an Größe zunehmen. Nach Abschluss des Wachstums tritt in der Regel eine Spontanheilung mit Sklerose der Läsion ein. Dies kann jedoch weit bis in das Erwachsenenalter beobachtet werden. Mit der Ausnahme der ossären Instabilität bei sehr großen Defekten bedürfen der fibröse Kortikalisdefekt und das NOF keiner spezifischen Therapie und sind daher klassische „leave-me-alone-lesions“.

\section{Fibröse Dysplasie}

Die fibröse Dysplasie (FD) imponiert histologisch als fibröses, spindelzelliges Bindegewebe, welches die Potenz zur Bildung von unregelmäßig geformtem Geflechtknochen besitzt. Primär geht die FD von spongiösem Knochengewebe aus, jedoch ist eine Affektion der Kortikalis möglich, was v.a. an der unteren 


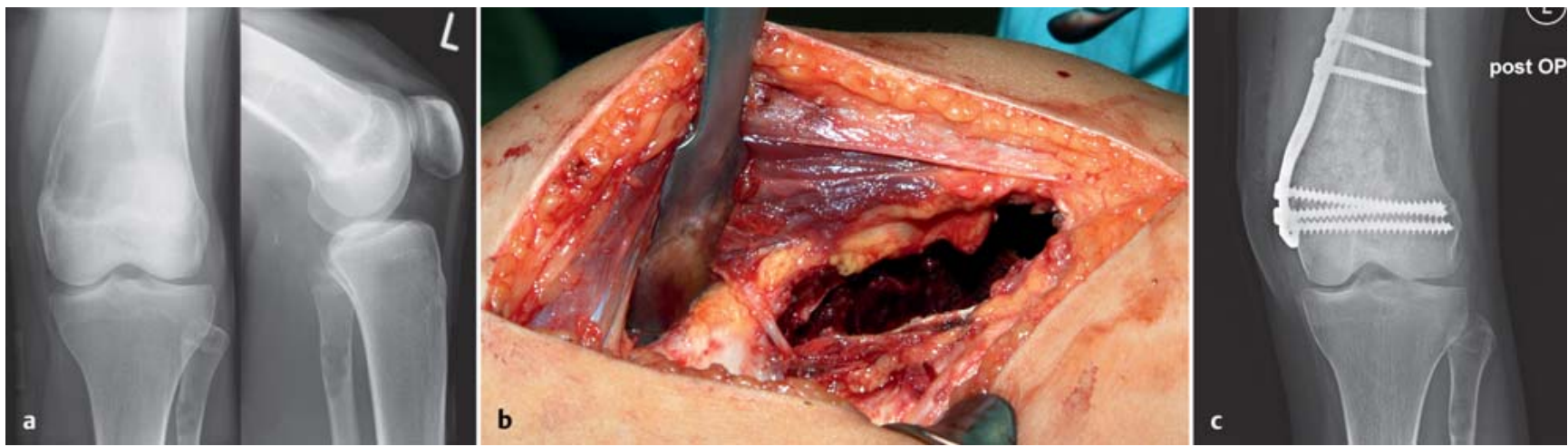

Abb. $\mathbf{5}$ a bis c Riesenzelltumor: Riesenzelltumor Condylus lateralis femoris: a konventionelles Röntgen, $\mathbf{b}$ intraläsionale Resektion durch Kürettage, c Augmentation durch Plattenosteosynthese nach Transplantation von allogener Spongiosa.

Extremität zu biomechanischen Problemen mit pathologischen Frakturen oder Achsabweichungen der langen Röhrenknochen führen kann. Eine maligne Entartung ist mit $<1 \%$ sehr selten. Die Therapie ist beschwerdeorientiert, wobei an der oberen Extremität eine konservative Behandlung favorisiert wird. Ziele einer operativen Maßnahme sind in erster Linie Herstellung von knöcherner Stabilität mit geeigneten Implantaten an der unteren Extremität. Eventuell ist eine Dekompression von Nerven und Gefäßen notwendig. Bisphosphonate beeinflussen den Krankheitsverlauf nicht, können jedoch Schmerzen lindern.

\section{Solitäre Knochenzyste}

Die einfache Knochenzyste wird häufig auch als juvenile oder solitäre Knochenzyste bezeichnet. Die Pathogenese ist unklar. Es handelt sich um keine neoplastische Läsion. Vielmehr wird eine reaktive Genese angenommen. Die einfache Knochenzyste besteht aus einer solitären, flüssigkeitsgefüllten Höhle, die von einer Membran umgeben ist. Die häufigste Lokalisation betrifft den proximalen Humerus und das proximale Femur. Die Zyste tritt im metaphysären Bereich auf. Sie wird überwiegend im Kindesund Jugendalter beobachtet. Jungen sind häufiger betroffen. Die Zyste führt zu einer Schwächung der Knochenstruktur, weshalb die überwiegende Anzahl der Patienten mit einer pathologischen Fraktur vorstellig werden. Instabilitätsschmerz ist ein weiteres Symptom bei großer Zyste. Die Zyste nimmt in der frühen Kindheit in der aktiven Phase deutlich an Größe zu. Im Alter von ca. 12 Jahren tritt die latente Phase ein. Aufgrund des begleitenden Längenwachstums rückt die Zyste näher in Schaftmitte. Nach Abschluss des Wachstums setzt ein langsamer Ossifikationsprozess ein, der überwiegend bereits mit Abschluss des 20 .
Lebensjahrs abgeschlossen ist. Die Diagnose kann in der Regel am konventionellen Röntgenbild gestellt werden. Es findet sich eine strahlentransparente Läsion mit einem scharf abgrenzbaren Rand. Die Kortikalis imponiert bleistiftdünn, ohne jedoch durchbrochen $\mathrm{zu}$ sein.

Die Behandlung der einfachen Knochenzyste wird kontrovers diskutiert, prinzipiell stellt sie eine „leave-me-alonelesion“ dar. Wir empfehlen in unserer Klinik eine „wait-and-see“-Strategie, da es sich um eine selbstlimitierende Erkrankung handelt. Frakturen in der Zyste heilen in der Regel unter konservativer Behandlung aus. Die Frakturheilung führt zu einer Beschleunigung der Ossifikation der Zyste. Nur bei rezidivierenden Frakturen ohne zunehmende Ossifikation sehen wir die Indikation zur therapeutischen Intervention. Das gängigste Verfahren stellt die (wiederholte) Installation von Kortison in die Zyste dar. Neuere Ansätze versuchen mit Stammzellen eine schnellere Ausheilung der Zyste zu erreichen. Die intraläsionale Resektion und Spongiosaauffüllung ist speziell in der aktiven Phase nicht zu empfehlen, da fast $50 \%$ rezidivieren.

\section{Aneurysmatische Knochenzyste}

Die aneurysmale Knochenzyste (AKZ) wird mit einer Häufigkeit von 2,5-6\% aller primären Knochentumoren angeben. Wie auch die juvenile Knochenzyste stellt sie kein Neoplasma dar. Es wird hier eine reaktive Komponente mit Veränderung der lokalen Zirkulation (z.B. nach Trauma) diskutiert. Die AKZ entsteht in der Hälfte der Fälle primär, in der anderen Hälfte der Fälle sekundär in Verbindung mit einem echten benignen oder malignen Neoplasma. Die AKZ kann in allen 3 Stadien auftreten. Die überwiegende Anzahl der Fälle wird vor dem
20. Lebensjahr entdeckt. Die Patienten werden in der Regel mit Schmerzen und einer harten Schwellung auffällig. Selten wird die AKZ im Rahmen von Frakturen diagnostiziert. Im Bereich der Wirbelsäule können Kompressionssyndrome die ersten Symptome für eine AKZ sein. Neben der Wirbelsäule sind v.a. die Metaphysen der langen Röhrenknochen Prädilektionsstellen der AKZ. Seltener tritt die AKZ im Bereich des Beckens oder der Skapula in Erscheinung. Das klassische radiologische Bild der AKZ besteht aus einer transparenten, expansiven Läsion mit einer eierschaldünnen äußeren reaktiven knöchernen Begrenzung. Diese ist im konventionellen Bild an einigen Stellen fehlend oder unterbrochen. Hier ist die Läsion lediglich vom Periost umgeben. Dies führt zum Bild einer Weichteilexpansion. Die AKZ ist septiert. Die Septen können sich im konventionellen Bild darstellen. Charakteristisch für die AKZ sind in der MRT wie auch in der CT sog. Fluid-Fluid-Levels. Innerhalb der Septen kommt es durch Sedimentierung des Zysteninhalts zum Bild der Flüssigkeit über der Flüssigkeit mit einer klaren geraden Trennlinie. Makroskopisch imponiert die AKZ mit multiplen, blutgefüllten, septierten Räumen, die von solidem Gewebe umgeben sind. Die wichtigsten Differenzialdiagnosen sind die solitäre Knochenzyste, der Riesenzelltumor und als ungünstigste Variante das teleangiektatische Osteosarkom. Die Behandlung des AKZ ist primär chirurgisch. Im Stadium 1 und 2 ist bei intraläsionaler Resektion die Rezidivrate zu vernachlässigen. Im Stadium 3 sollte bei intraläsionaler Resektion ein Adjuvans genutzt oder aber eine marginale oder weite Resektion durchgeführt werden. Operativ schlecht zugängliche Läsionen können durch (wiederholte) Embolisationen teils erfolgreich therapiert werden, dies ist insbesondere im Bereich der Wirbelsäule 
als therapeutische Option in Erwägung zu ziehen (Abb. 2).

\section{Riesenzelltumor}

Der Riesenzelltumor des Knochens (Synonym: Osteoklastom) ist ein lokal aggressiv wachsendes Neoplasma. Obwohl er prinzipiell in jeder Lokalisation auftreten kann, besteht eine klare Prädilektion im Bereich der Epiphyse der großen langen Röhrenknochen. Der überwiegende Teil imponiert als Stadium-2-Läsion, ca. ein Drittel als Stadium-3-Läsion. Unabhängig vom Stadium können in 1$2 \%$ der Patienten Lungenmetastasen gefunden werden. Diese sind ebenfalls als benigne anzusehen. Das Prädilektionsalter liegt zwischen 20 und 40 Jahren, Riesenzelltumoren im wachsenden Skelett sind sehr selten und sollten Anlass sein, die Diagnose zu überprüfen. Riesenzelltumoren vor Abschluss des Wachstums befallen typischerweise die Metaphyse. In seltenen Fällen kann ein multilokulärer Befall gefunden werden. Klinische Symptome sind Schmerz und Schwellung, häufig begleitet von einer Bewegungseinschränkung des Gelenks.

Der Riesenzelltumor zeigt konventionell radiologisch das typische Bild einer landkartenartigen, osteolytischen Läsion. Die Läsion ist strahlentransparent. Es findet sich ein scharfer Rand, jedoch ohne Sklerose. Die Lokalisation ist häufig exzentrisch. Expansives Wachstum ist möglich. Eine Periostreaktion fehlt gewöhnlicherweise. Im aggressiven Stadium fehlt eine innere Trabekelbildung. Eine Destruktion der Kortikalis mit Weichteilkomponente ist typisch. Die Kortikalisdestruktion kann am besten in der CT nachgewiesen werden. Die MRT hat ih- ren klaren Vorteil in der Beurteilung der extraossären Komponente, speziell bei Verdacht auf eine Gelenkinfiltration. Eine Szintigrafie ist zum Ausschluss eines multilokulären Befalls oder auch von Skip Lesions empfehlenswert.

Die Behandlung des Riesenzelltumors ist chirurgisch. Die intraläsionale Resektion erscheint aufgrund des lediglich lokal aggressiven Charakters des Tumors gerechtfertigt. Für eine erfolgreiche Operation ist ein ausreichend großes Knochenfenster entscheidend, über das eine komplette Inspektion und Kürettage aller Ränder möglich ist. Die Rezidivrate ist jedoch hoch, sodass im Verlauf eine Rekürettage oder aber eine weite Resektion notwendig wird. Bei intraläsionaler Resektion sollten adjuvante Maßnahmen zur Erweiterung des Resektionsrands erfolgen. Die Implantation von PMMA kann aufgrund der häufig sehr subchondralen Lage zu Gelenkschmerzen führen. Zur Minderung ist die Implantation von einigen Millimetern subchondraler Spongiosa empfohlen (Abb. 5).

\section{Schlussfolgerung}

Die korrekte Behandlung benigner Knochentumoren setzt eine detaillierte und differenzierte Diagnostik voraus, die in der Zusammenschau aus Anamnese, Klinik und Bildgebung erfolgt. Bei radiologisch unklaren Befunden ist neben einer Verlaufskontrolle in einigen Fällen eine Biopsie erforderlich.

Die Therapie der gutartigen Tumoren des Knochens ist abhängig von der Tumorentität, der Lokalität, der klinischen Symptomatik oder einer evtl. knöchernen Sta- bilitätsgefährung. Häufig sind gutartige Tumoren klinisch inapparente Zufallsbefunde und bedürfen keiner konkreten Behandlung - sog. „leave-me-alone-lesions“.

\section{Literatur}

${ }^{1}$ Fletcher CDM, Unni K, Mertens K, eds. WHO Classification of Tumours. Pathology and Genetics of Tumours of Soft Tissue and Bone. Lyon: IARC Press; 2002

2 Rodl $R$, Gotze C. [Fibrous dysplasia]. Orthopade 2008; 37: 49-55

3 Streitbuerger A, Hardes J, Gebert C et al. [Cartilage tumours of the bone. Diagnosis and therapy]. Orthopade 2006; 35: 871-881

${ }^{4}$ Freyschmidt J, Ostertag H, Jundt G. Textbook of Bone Tumors. Berlin, Heidelberg: Springer; 2010

${ }^{5}$ Campanacci M. Textbook of Bone and Soft Tissue Tumors: Clinical Features, Imaging, Pathology and Treatment. Wien, New York: Springer; 1999

${ }^{6}$ Schajowicz F, Sissons HA, Sobin LH. The World Health Organization's histologic classification of bone tumors. A commentary on the second edition. Cancer 1995; 75: 1208-1214

7 Dahlin DC. Bone tumors. 3rd ed. Springfield: Thomas; 1978

8 Schajowicz F. Textbook of tumors and tumorlike lesions of bone. 2nd ed. Berlin, Heidelberg: Springer; 1994

\section{Ulf Brunnemer \\ Assistenzarzt}

Prof. Dr. med. Christian Krettek

Direktor der Klinik

Prof. Dr. med. Thomas Gösling

Ltd. Oberarzt der Klinik

Unfallchirurgische Klinik Medizinische Hochschule Hannover Carl-Neuberg-Straße 1 30625 Hannover

brunnemer.ulf@mh-hannover.de 